\title{
Private entanglement over arbitrary distances, even using noisy apparatus
}

\author{
Hans Aschauer and Hans J. Briegel \\ Sektion Physik, Ludwig-Maximilians-Universität, \\ Theresienstr. 37, D-80333 München, Germany
}

\begin{abstract}
We give a security proof of quantum cryptography based entirely on entanglement purification. Our proof applies to all possible attacks (individual and coherent). It implies the security of cryptographic keys distributed with the help of entanglementbased quantum repeaters. We prove the security of the obtained quantum channel which may not only be used for quantum key distribution, but also for secure, albeit noisy, transmission of quantum information.
\end{abstract}

Quantum cryptography (QC) promises the security of data transmission against any eavesdropping attack allowed by the laws of physics. The first QC protocol was described by Bennett and Brassard as early as 1984 [1]. Later, in 1991 Ekert presented a scheme based on Bell's theorem [2]. Though the security of these protocols is easy to prove under ideal conditions, a lot of work has been spent to prove the security under realistic circumstances. In all QC protocols, a possible eavesdropper is identified because of the disturbance that he or she introduces when trying to gain information about a quantum state that is transmitted. The problem is that every quantum channel introduces innocuous noise itself, which cannot, in principle, be distinguished from noise introduced by an eavesdropper. For that reason, a proof of unconditional security of QC has to assume that all noise in the channel is due to the interference of an eavesdropper.

Two different techniques have been developed to deal with these difficulties: Classical privacy amplification allows the eavesdropper to have partial knowledge about the raw key built up between the communicating parties Alice and Bob. From the raw key, a shorter key is "distilled" about which Eve has vanishing (i.e. exponentially small in some chosen security parameter) knowledge. Despite of the simple idea, proofs taking into account all eavesdropping attacks allowed by the laws of quantum mechanics have shown to be technically involved [3, 4, 5]. Recently, Shor and Preskill [6] have given a simpler physical proof relating the ideas in [3, 5] to quantum error correcting codes [7, 8]. Quantum privacy amplification (QPA) [9], on the other hand, employs an entanglement purification [10, 11] protocol that eliminates any entanglement with an eavesdropper by creating a few perfect EPR pairs out of many imperfect (or impure) EPR pairs. In principle, this method guarantees security against any eavesdropping attack. However, the problem is that the QPA protocol assumes ideal quantum operations. In reality, these operations are themselves subject to noise. As shown in 112, 13, 14, there is an upper bound $F_{\max }$ for the achievable fidelity of EPR pairs which can be distilled using noisy apparatus. A priori, there is no way to be sure that there is no residual entanglement with an eavesdropper. This problem could be solved if Alice and Bob had fault tolerant quantum computers at their disposal, which could then be used to reduce the noise of the apparatus to any desired level. This was an essential assumption in the security proof given by Lo and Chau [15].

In this paper, we show that the standard two-way entanglement purification protocols alone, with some minor modifications to accomodate certain security aspects which will be 
discussed below, can be used to efficiently establish a perfectly private quantum channel, even when both the physical channel connecting the parties and the local apparatus used by Alice and Bob are noisy. This is of particular interest because, as we show, the security threshold for the noise-level of the apparatus practically coincides with the purification threshold, so that the methods used for long-distance quantum communication, using entanglementpurification-based quantum repeaters [12, 13] can be used for secure quantum communication without any further requirements. In particular, no fault tolerant quantum computers are required. This goal is achieved by proving that the final state of the protocol factorizes into a product state of the eavesdropper on one side, and Alice, Bob and their laboratories (apparatuses) on the other side. Colloquially speaking, we prove that Eve is factored out under the action of the purification protocol, i. e. the finite fidelity at the end of the protocol is only due to entanglement with the apparatus. Our proof applies to all possible attacks (individual, collective, and coherent) and can be utilized directly in long-distance quantum communication. Different from existing work, we (i) prove the security of the entire quantum channel, (ii) do not require fault tolerant quantum computers, and (iii) our results have practical relevance, as the accuracy of the apparatus used by Alice and Bob may be about two orders of magnitude lower than the threshold accuracy for fault tolerant quantum computers [12, 16].

The scenario is the following. Initially, Alice and Bob share a numbered ensemble of $2 N$ qubits $\left\{\left(a_{1}, b_{1}\right), \ldots,\left(a_{N}, b_{N}\right)\right\}, N$ qubits on each side, where $N$ is large. Most generally, the state they obtain can be written in the form

$$
\rho_{A B}=\sum_{\substack{\mu_{1} \ldots \mu_{N} \\ \mu_{1}^{\prime} \ldots \mu_{N}^{\prime}}} \alpha \mu_{1}^{\mu_{1} \ldots \mu_{N}} \mu_{N}^{\prime}\left|\mathcal{B}_{\mu_{1}}^{\left(a_{1} b_{1}\right)} \cdots \mathcal{B}_{\mu_{N}}^{\left(a_{N} b_{N}\right)}\right\rangle\left\langle\mathcal{B}_{\mu_{1}^{\prime}}^{\left(a_{1} b_{1}\right)} \cdots \mathcal{B}_{\mu_{N}^{\prime}}^{\left(a_{N} b_{N}\right)}\right|
$$

where $\left|\mathcal{B}_{\mu_{j}}^{\left(a_{j} b_{j}\right)}\right\rangle, \mu_{j}=00,01,10,11$ denote the 4 Bell states associated with the two particles $a_{j}$ and $b_{j}$. Specifically, $\left|\mathcal{B}_{00}\right\rangle \equiv\left|\Phi^{+}\right\rangle=(|00\rangle+|11\rangle) / \sqrt{2},\left|\mathcal{B}_{01}\right\rangle \equiv\left|\Psi^{+}\right\rangle=(|01\rangle+|01\rangle) / \sqrt{2}$, $\left|\mathcal{B}_{10}\right\rangle \equiv\left|\Phi^{-}\right\rangle=(|00\rangle-|11\rangle) / \sqrt{2},\left|\mathcal{B}_{11}\right\rangle \equiv\left|\Psi^{-}\right\rangle=(|01\rangle-|10\rangle) / \sqrt{2}$. The qubits have been distributed through some noisy channel, which may also include repeater stations involving additional qubits [12]. In general, (1) will be an entangled state of $2 N$ particles, which allows for the possibility of so-called coherent attacks [17]. This state may be used to establish a perfectly secret quantum channel, given that there is one Bell state, say $\left|\mathcal{B}_{00}\right\rangle$, such that $\left\langle\mathcal{B}_{00}^{\left(a_{j} b_{j}\right)}\left|\rho_{a_{j} b_{j}}\right| \mathcal{B}_{00}^{\left(a_{j} b_{j}\right)}\right\rangle>F_{\text {min }}>1 / 2$ for the reduced density operator of every pair of qubits $\left(a_{j}, b_{j}\right)$, where the exact value of $F_{\min }$ depends on the noise parameters of Alice's and Bob's apparatus [12, 14].

Upon reception of all pairs, Alice and Bob apply the following protocol to them. Note that steps 1 and 2 are only applied once, while steps 3, 4, and 5 are applied recursively.

Step 1: On each pair of particles $\left(a_{j}, b_{j}\right)$, they apply randomly one of the four bi-lateral Pauli rotations $\sigma_{k}^{\left(a_{j}\right)} \otimes \sigma_{k}^{\left(b_{j}\right)}$, where $\mathrm{k}=0,1,2,3$.

Step 2: Alice and Bob randomly renumber the pairs, $\left(a_{j}, b_{j}\right) \rightarrow\left(a_{\pi(j)}, b_{\pi(j)}\right)$ where $\pi(j)$, $j=1, \ldots, N$ is a random permutation.

Steps 1 and 2 are required in order to treat correlated pairs correctly. Note that steps 1 and 2 would also be required - as "preprocessing" steps - for the ideal protocol [9], if one requires that the protocol converges for arbitrary states of the form (1) to an ensemble of pure EPR states. While in [9] it is possible to check whether or not the protocol converges to the desired pure state, by measuring the fidelity of some of the remaining pairs, this is not possible when imperfect apparatus is used. Since the maximum attainable fidelity $F_{\max }$ 
is smaller than unity, there is no known way to exclude the possibility that the non-ideal fidelity is due to correlations between the initial pairs. In both steps Alice and Bob discard the information which of the rotations and permutations, respectively, were chosen by their random number generator. Thus they deliberately loose some of the information about the ensemble which is still available to Eve. After step 1, their knowledge about the state is summarized by the density operator

$$
\tilde{\rho}_{A B}=\sum_{\mu_{1} \ldots \mu_{N}} p_{\mu_{1} \ldots \mu_{N}}\left|\mathcal{B}_{\mu_{1}}^{\left(a_{1} b_{1}\right)} \cdots \mathcal{B}_{\mu_{N}}^{\left(a_{N} b_{N}\right)}\right\rangle\left\langle\mathcal{B}_{\mu_{1}}^{\left(a_{1} b_{1}\right)} \cdots \mathcal{B}_{\mu_{N}}^{\left(a_{N} b_{N}\right)}\right|
$$

which corresponds to a classically correlated ensemble of pure Bell states. Since the purification protocol that they are applying in the following steps maps Bell states onto Bell states, it is statistically consistent for Alice and Bob to assume after step 1 that they are dealing with a (numbered) ensemble of pure Bell states, where they have only limited knowledge about which Bell state a specific pair is in. The fact that the pairs are correlated means that the order in which they appear in the numbered ensemble may have some pattern, which may have been imposed by Eve or by the channel itself. By applying step 2, Alice and Bob (i) deliberately ignore this pattern and (ii) randomize the order in which the pairs are used in the subsequent purification steps. For all statistical predictions made by Alice and Bob, they may consistently describe the ensemble by the density operator [20]

$$
\tilde{\tilde{\rho}}_{A B}=\left(\sum_{\mu} p_{\mu}\left|\mathcal{B}_{\mu}\right\rangle\left\langle\mathcal{B}_{\mu}\right|\right)^{\otimes N} \equiv\left(\rho_{a b}\right)^{\otimes N}
$$

in which the $p_{\mu}$ describe the probability with which each pair is found in the Bell state $\left|\mathcal{B}_{\mu}\right\rangle$. At this point, Alice and Bob have to make sure that $p_{00} \equiv F>F_{\min }$ for some minimum fidelity $F_{\min }>1 / 2$, which they can do by statistical tests on a certain fraction of the pairs. Next, Alice and Bob apply one of the standard purification protocols as described in [9, 10]. For simplicity, we concentrate on the protocol given in [9]; for other recurrence protocols, a similar proof could be given [21]. The protocol uses these steps:

Step 3: Bi-lateral rotations $1 / 2\left(\mathbf{1}^{(a)}-i \sigma_{x}^{(a)}\right) \otimes\left(\mathbf{1}^{(b)}+i \sigma_{x}^{(b)}\right)$ are applied to all pairs $(a, b)$. Step 4: To all pairs of pairs a bi-lateral CNOT operation (BCNOT) is applied.

Step 5: The target pair of the BCNOT operation is measured on both sides in $z$-direction. If the measurement results coincide, the control pair is kept, otherwise it is discarded.

Since Alice and Bob use imperfect apparatus, it has been shown [12, 14] that these protocols converge towards a mixed-state ensemble $\rho_{a b}^{(\infty)}$ with a maximum attainable fidelity $F_{\max }<1$. If the fidelity of the local operations is moderate, the value of $F_{\max }$ could be quite low $(80 \%$, as an example).

In the following we will show that, despite of such a poor attainable fidelity, Alice and Bob may happily proceed to apply the purification protocol to establish a secure quantum channel [22]. We show that, as $F \rightarrow F_{\max }$, the entanglement of the ensemble with the eavesdropper is reduced exponentially fast with the number of purification steps. In each step of the protocol, we assume that the apparatus they use introduces errors described by the following map 23]

$$
\rho_{A B} \rightarrow \sum_{\mu, \nu=0}^{3} f_{\mu \nu} \sigma_{\mu}^{(a)} \sigma_{\nu}^{(b)} \rho_{A B} \sigma_{\mu}^{(a)} \sigma_{\nu}^{(b)},
$$


where $a$ and $b$ denote the qubits which are acted upon locally. The $f_{\mu \nu}$ can be interpreted as the joint probability that the Pauli rotations $\sigma_{\mu}$ and $\sigma_{\nu}$ are applied to qubits $a$ and $b$, respectively. Eq. (4) includes, for an appropriate choice of the coefficients $f_{\mu \nu}$, the one and two qubit depolarizing channel and combinations thereof, as studied in [12, 13], but is more general.

It is possible to include the laboratories degrees of freedom in the description. Noise of the form (\#) can be attributed to some interaction with the apparatus, which is described by a map

$$
|E\rangle_{L}|\psi\rangle_{A B} \rightarrow \sum_{\mu, \nu=0}^{3}\left|e_{\mu \nu}\right\rangle_{L} \sigma_{\mu}^{(a)} \sigma_{\nu}^{(b)}\left|\psi^{(a b)}\right\rangle .
$$

This map explicitly accounts for the state of the apparatus before and after the interaction. The states $\left|e_{\mu \nu}\right\rangle$ are pairwise orthogonal and have the norm $\left\langle e_{\mu \nu} \mid e_{\mu \nu}\right\rangle=f_{\mu \nu}$. It is important to note that the laboratory degrees of freedom $\left|e_{\mu \nu}\right\rangle$ can, in principle, be identified in any physical environment that generates noise of the form (4), if the specific interaction Hamiltonian is known.

For our purpose, however, the physical details of the environment are of no concern, and we may replace the real process by the following scenario, where both Alice and Bob have a "little demon" (L) in the laboratory. For simplicity, we concentrate on the demon in Alices laboratory only. Note that the generalisation to noise in both labs is trivial. Before every purification step, the demon applies randomly one of the sixteen rotations $\sigma_{\mu}^{(a)} \otimes \sigma_{\nu}^{(b)}$ to the qubits involved it this step, and keeps a record of which rotations he chose. For example, in the case of uncorrelated white noise (depolarizing channel), it leaves each qubit in its state $\left(\sigma_{0} \equiv I\right)$ with some probability $f_{0}$, but rotates its state by $\sigma_{j}$ with equal probabilities $f_{j}=\frac{1-f_{0}}{3}$.

By doing this, the demon may accumulate a record of all errors in the history of each qubit throughout the process. Instead of keeping track of this growing list, he updates in each purifications step a single flag $\phi \equiv(i j)$ that is associated with each of the pairs. The aim of the error flag is to keep the information required for "undoing" the random rotations that occured in the history of each pair. Note that, while this can be done trivially for unitary networks, the situation is quite different with the QPA destillation protocol, which includes measurements. For the proof, we show that there exists a flag update function as discussed below - which enables the lab demon at any time of the protocol, to assign each of the pairs to one out of four subensembles of the total ensemble of pairs and to keep track of each of these subensembles seperately. Technically, the flag consists of two classical bits, called the error phase bit $i$ and the error amplitude bit $j$. The update is done in the following way: If a $\sigma_{x}\left(\sigma_{z}, \sigma_{y}\right)$ error occurs, L inverts the error amplitude bit (error phase bit, both error bits). Whenever Alice and Bob agree publicly to keep a control pair $P_{1}$ (because of coinciding measurement outcomes on the target pair $P_{2}$, see step 5 of the protocol), L calculates a function of the error flags of $P_{1}$ and $P_{2}$ that updates the flag of $P_{1}$. The function is shown in table [1. Note that the error flag which belongs to a pair is, by construction, only a function of the error records. It is important to realize that, what the lab demon is doing is not quantum error correction, as he is not applying any correction operation on the qubits in the course of the entire protocol. Instead of calculating the flags during the run of the protocol, they could equally be calculated after the protocol is finished.

As mentioned earlier, at each purification step, the lab demon divides the total ensemble into four subensembles $\rho_{\mathrm{AB}}^{(i j)}$ corresponding to the value $(i j)$ of the error flag. Initially, before 


\begin{tabular}{|l|llll|}
\hline \hline & $(00)$ & $(01)$ & $(10)$ & $(11)$ \\
\hline$(00)$ & $(00)$ & $(00)$ & $(00)$ & $(10)$ \\
$(01)$ & $(00)$ & $(01)$ & $(11)$ & $(00)$ \\
$(10)$ & $(00)$ & $(11)$ & $(01)$ & $(00)$ \\
$(11)$ & $(10)$ & $(00)$ & $(00)$ & $(00)$ \\
\hline \hline
\end{tabular}

TABLE I: The value (phase error,amplitude error) of the updated error flag of a pair that is kept after a QPA step, given as a function of the error flags of $P_{1}$ and $P_{2}$ (left to right and top to bottom, respectively).

the QPA protocol starts, he assigns some random or fixed values to the labels, while the subensembles are all in the same state. That is, the error flags and the states of the pairs are initially completely uncorrelated. It is noteworthy that Bell diagonality of the states $\rho_{\mathrm{AB}}^{(i j)}=A^{(i j)}\left|\mathcal{B}_{00}\right\rangle\left\langle\mathcal{B}_{00}\left|+B^{(i j)}\right| \mathcal{B}_{11}\right\rangle\left\langle\mathcal{B}_{11}\left|+C^{(i j)}\right| \mathcal{B}_{01}\right\rangle\left\langle\mathcal{B}_{01}\left|+D^{(i j)}\right| \mathcal{B}_{10}\right\rangle\left\langle\mathcal{B}_{10}\right|$ is preserved. This is due to the fact that all operations in the protocol map Bell states onto Bell states.

In the following, we analyse the purification process in terms of these four different subensembles $\rho_{\mathrm{AB}}^{(i j)}$. In total, we have to keep track of 16 coefficients that occur in the expansion of each of the $\rho_{\mathrm{AB}}^{(i j)}$ in the Bell basis. These coefficients after the $(n+1)$-th QPA step are functions of the coefficients after the $n$-th QPA step:

$$
\begin{aligned}
A_{n}^{(00)} & \rightarrow A_{n+1}^{(00)}\left(A_{n}^{(00)}, A_{n}^{(01)}, \ldots, D_{n}^{(11)}\right), \\
A_{n}^{(01)} & \rightarrow A_{n+1}^{(01)}\left(A_{n}^{(00)}, A_{n}^{(01)}, \ldots, D_{n}^{(11)}\right), \\
& \vdots \\
D_{n}^{(11)} & \rightarrow D_{n+1}^{(11)}\left(A_{n}^{(00)}, A_{n}^{(01)}, \ldots, D_{n}^{(11)}\right) .
\end{aligned}
$$

The explicit form of the 16 recurrence relations (6) can be given, but they are rather lengthy. They imply a reduced set of 4 recurrence relations for the quantities $A_{n}=$ $\sum_{i j} A_{n}^{(i j)}, \ldots, D_{n}=\sum_{i j} D_{n}^{(i j)}$ which describe the evolution of the total ensemble under the purification protocol. For $n \rightarrow \infty$, these quantities converge towards a fixpoint $\left(A_{\infty}, B_{\infty}, C_{\infty}, D_{\infty}\right)$ where $A_{\infty}=F_{\max }$ is the maximal attainable fidelity [13]. Different from the fidelity $F_{n} \equiv A_{n}$, we define the conditional fidelity $F_{n}^{\text {cond }}=A_{n}^{(00)}+B_{n}^{(11)}+C_{n}^{(01)}+D_{n}^{(10)}$. This is the fidelity of the ensemble that Alice and Bob could attain, if the lab demon disclosed the error flags (or, for that matter, only the history of the random rotations, from which the flags can be calculated): Depending on the error flag of a pair, Alice could then choose a local rotation that transforms the pair into the Bell state $\left|\mathcal{B}_{00}\right\rangle$ with probability $F^{\text {cond }}$.

Evaluation of the recurrence relation yields that there are three different regimes of noise parameters: In the high-noise regime (low values of $f_{00}$ ), no purification is possible; the protocol converges to completely depolarized pairs. In the low-noise regime (high values of $\left.f_{00}\right)$, the protocol purifies and the conditional fidelity converges to unity: the protocol is in the security regime. Between these two regimes, just above the purification threshold, there exists a very narrow third regime: The protocol purifies, while the conditional fidelity does not converge to unity. It is not known whether or not secure communication is possible in this regime. For the depolarizing channel, for example, the intermediate regime is contained in the interval $f_{0} \in(0.8983,0.8988)$, while the security regime covers the entire interval 


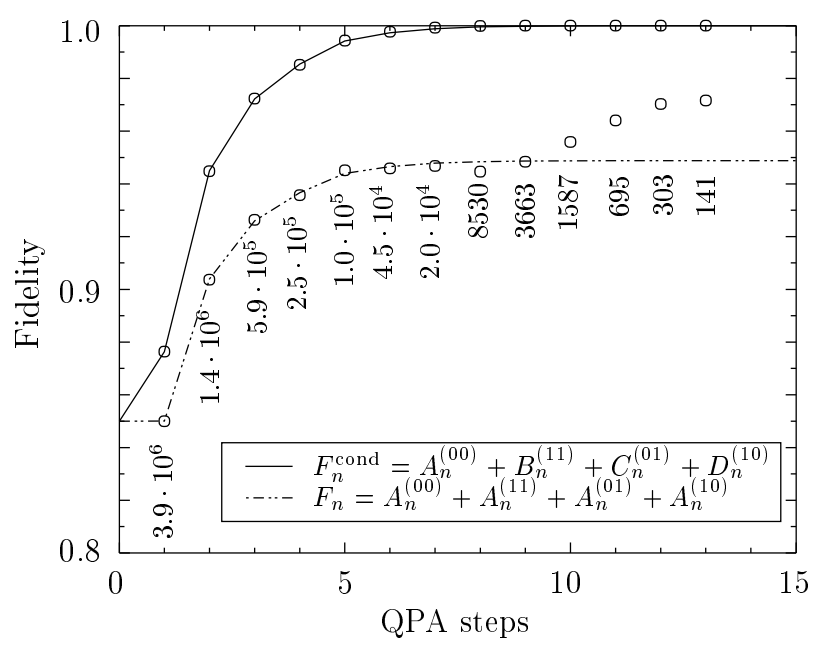

FIG. 1: The fidelities $F$ and $F_{\text {cond }}$ as a function of the number of steps in the QPA protocol (analytical results (lines) and Monte Carlo simulation (circles)). For the calculation, one and twoqubit white noise with a noise fidelity of $97 \%$ has been assumed. The Monte Carlo simulation was started with $10^{7}$ pairs; the numbers indicate how many pairs are left after each step of the purification protocol. This decreasing number is the reason for the increasing fluctuations around the analytical curves.

$f_{0} \in[0.8988,1]$. The security regime thus coincides, for all practical purposes, with the purification regime, but it is interesting to see that these regimes are not strictly identical. It shows that the process of factorization is, in the situation of imperfect apparatus, not trivially connected to the process of purification. More details about these regimes will be published elsewhere. When the protocol is in the security regime, both the fidelity $F_{n}$ and the conditional fidelity $F_{n}^{\text {cond }}$ reach their respective fixpoints exponentially fast with the same exponents (see Fig. 2). From this it follows that there exists a polynomial relation between the resources used in the purification process (number of initial pairs) and the security parameter $1-F^{\text {cond }}$. All results obtained from the evaluation of the recurrence relations (6) were also checked with the help of Monte Carlo simulations, in which the QPA protocol was applied to typical ensembles of Bell states.

Our results imply that the error flags and the states of the subensembles become strictly correlated during execution of the purification protocol: The subensemble $(i j)$ ends in the state $\left|\mathcal{B}_{i j}\right\rangle$. In other words, the "little demon" has acquired complete knowledge about the states of all pairs after sufficiently many purifications steps; the system consisting of the pairs and the lab is thus in a pure state. Now the same argument as in [9] applies: a system in a pure state cannot be entangled with any other system - any eavesdropper is factored out, as his or her entanglement with the pairs is lost.

This proof can be extended to more general noise models if a slightly modified protocol is used, where step 1 is repeated after every distillation round [24. This effectively regularises any type of local noise process to a process of the type (4) that conserves the Bell diagonality of the ensemble, for which we can apply the lab-demon interpretation [25].

The fact that the security regime of the protocol almost coincides with the purification regime is of strong practical interest because it implies that EPR pairs distributed over long distances with quantum repeaters can be used for secure quantum communication without 


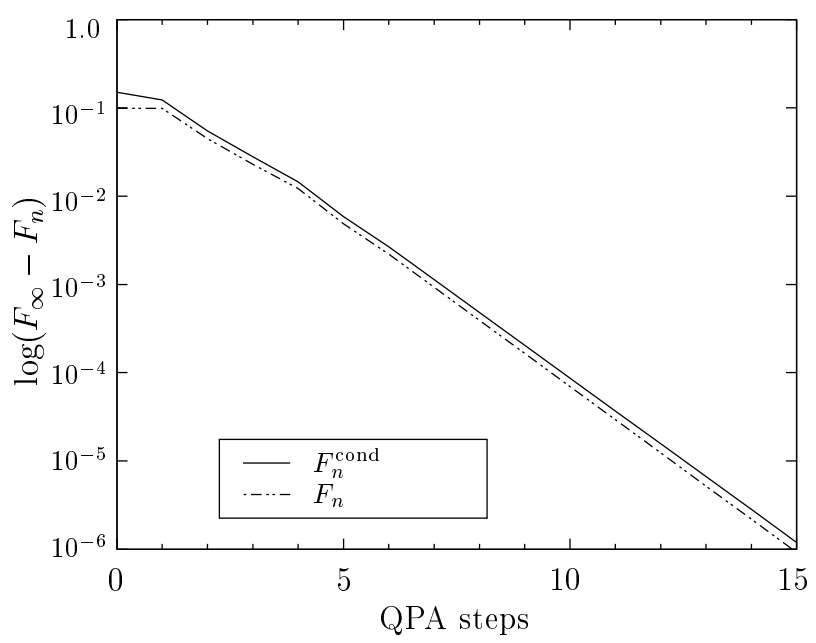

FIG. 2: $F_{\infty}-F_{n}$ and $1-F_{n}^{\text {cond }}$ plotted logarithmically against the purification step $n$. The parameters are the same as in Fig. 1.

any additional effort [26].

To summarize, Alice and Bob obtain, with the help of a standard entanglement purification protocol, entangled EPR pairs. These pairs have a limited fidelity $F \lesssim F_{\max }<1$ which depends on the noise introduced by local operations in their laboratory. Alice and Bob may nevertheless use these pairs for secure quantum- or classical communication, e. $g$. teleportation [18] or key distribution. At this stage, no further security tests are necessary. Since we have shown that there exists no residual entanglement with an eavesdropper, they may use all the pairs for the key! While there may be a significant error rate in the message, Alice and Bob are allowed to apply classical error correction to the transmitted message without disclosing any valuable information to Eve.

\section{Acknowledgments}

We thank C. H. Bennett, A. Ekert, L. Hardy, H. Inamori, N. Lütkenhaus, R. Raussendorf, A. Schenzle and H. Weinfurter for valuable discussions. We are grateful to G. Giedke, N. Lütkenhaus, and H.-K. Lo for constructive remarks on the manuscript. This work has been supported in part by the Schwerpunktsprogramm QIV of the DFG.

[1] C. H. Bennett and G. Brassard, in Proceedings of IEEE International Conference on Computers, Systems and Signal Processing, Bangalore, India (IEEE, New York, 1985), pp. 175-179.

[2] A. Ekert, Phys. Rev. Lett. 67, 661 (1991).

[3] E. Biham et al., in Proceedings of the Thirty-Second Annual ACM Symposium on Theory of Computing (ACM Press, New York, 2000), pp. 715-724.

[4] H. Inamori, quant-ph/0008064 (unpublished).

[5] D. Mayers, in Advances in Cryptology-Proceedings of Crypto '96 (Springer, New York, 1996), pp. 343-357. 
[6] P. W. Shor and J. Preskill, Phys. Rev. Lett. 85, 441 (2000).

[7] A. R. Calderbank and P. Shor, Phys. Rev. A 54, 1098 (1996).

[8] A. M. Steane, Phys. Rev. Lett. 77, 793 (1996).

[9] D. Deutsch et al., Phys. Rev. Lett. 77, 2818 (1996).

[10] C. H. Bennett et al., Phys. Rev. Lett. 76, 722 (1996).

[11] C. H. Bennett et al., Phys. Rev. A 54, 3824 (1996).

[12] H.-J. Briegel et al., Phys. Rev. Lett. 81, 5932 (1998).

[13] W. Dür et al., Phys. Rev. A 59, 169 (1999).

[14] G. Giedke et al., Phys. Rev. A 59, 2641 (1999).

[15] H.-K. Lo and H. F. Chau, Science 283, 2050 (1999).

[16] For a review, see e.g. J. Preskill, quant-ph/9712048.

[17] J. I. Cirac and N. Gisin, Phys. Lett. A 229, 1 (1997).

[18] C. H. Bennett et al., Phys. Rev. Lett. 70, 1895 (1993).

[19] J.-W. Pan et al., Nature 410, 1067 (2001).

[20] While, strictly speaking, this equality holds only for $N \rightarrow \infty$, the subsequent arguments also hold for the exact but more complicated form of (3) for finite $N$.

[21] Note that for noisy local operations the hashing protocol, which requires Alice and Bob to apply a large number of CNOT operations in every distillation step, usually performs much worse that the recurrence protocols. The reason for this lies in the fact that the noise introduced with every CNOT operation accumulates and rapidly shatters the potential information that could ideally be gained from the parity measurement.

[22] The fact that already the rotations used in steps 1 and 2 will be subject to noise is immaterial. As no measurements are performed, all such noise may be entirely attributed to the channel.

[23] We implicitly assume that Alice and Bob can trust their apparatus. Similar as in the work of Mayers and Yao, quant-ph/9809039, Alice and Bob need not trust the apparatus that produces the qubits. In the scenario considered in the present paper the quantum EPR source may be entirely under external control (e.g. repeater stations) and is thus not trusted at all. Alice and Bob merely check, before step 3 of the protocol, the fidelity of the ensemble.

[24] We are grateful to C. H. Bennett for pointing out this possibility.

[25] Here, it is however required that Alice and Bob are able to perform one-qubit rotations used in step 1 well enough to keep the evolution Bell-diagonal.

[26] The relevance of this result is underlined by a recent proposal by Pan et al. [19], who describe a scheme of EPP using only optical elements, with which one would be able to reach the desired error-threshold. 